Research Article

\title{
A Descriptive Study to Assess the Prevalence of Compassion Fatigue, Burnout and Compassion Satisfaction among Staff Nurses Working in Selected Hospitals of Gautam Buddh Nagar, Uttar Pradesh, India
}

\author{
Krishna Kumari', Lekha Bist ${ }^{2}$ \\ ${ }^{1}$ Lecturer, ${ }^{2}$ Principal, Kailash Institute of Nursing and Paramedical Sciences, Greater Noida, Uttar Pradesh, India. \\ DOI: https://doi.org/10.24321/2455.9318.202021
}

\section{I $\quad \mathbf{N} \quad \mathbf{F} \quad \mathbf{O}$}

E-mail Id:

krishna.ku1227@gmail.com

Orcid Id:

https://orcid.org/0000-0003-4150-390X

How to cite this article:

Kumari K, Bist L. A Descriptive Study to Assess the Prevalence of Compassion Fatigue, Burnout and Compassion Satisfaction among Staff Nurses Working in Selected Hospitals of Gautam Buddh Nagar, Uttar Pradesh, India. Int J Nurs Midwif Res 2020; 7(3): 16-23.

Date of Submission: 2020-11-03

Date of Acceptance: 2021-02-25

\section{$\begin{array}{llllllll}\mathbf{A} & \mathbf{B} & \mathbf{S} & \mathbf{T} & \mathbf{R} & \mathbf{A} & \mathbf{C} & \mathbf{T}\end{array}$}

\begin{abstract}
Introduction: Compassion fatigue among midwives has gained interest over the past decade. Midwives in general are exposed to the risk of Compassion Fatigue (CF), Burnout (BO) and low levels of Compassion Satisfaction (CS). Aim of this study was to assess the prevalence of compassion fatigue, burnout, and compassion satisfaction among staff nurses working in selected hospital of Gautam Buddh Nagar. Objectives of this study were to assess the prevalence of compassion fatigue, burnout and compassion satisfaction among staff nurses in selected hospitals of Gautam Buddh Nagar and to determine association between compassion fatigue, burnout, compassion satisfaction and selected demographic variables.
\end{abstract}

Methods: A non-experimental descriptive survey design was used for the study. Sample size consisted of 50 midwives by purposive sampling technique. Data was collected by administering the standardized questionnaire i.e. Professional Quality of Life Scale (Pro QOL) by interview technique. Data was analyzed using descriptive and inferential statistics.

Result: Result revealed that majority of midwives 43 (86\%) had average level of compassion fatigue, 33 (66\%) midwife nurses had average level of burnout, only 16 (32\%) midwife nurses had high level of compassion satisfaction. The prevalence of compassion satisfaction mean score was 38.12 , mean percent score was 76.24 , median score was 39 , and SD score was 5.92 among the midwives. There was no significant association between the compassion fatigue and demographic variables but significant association between the burnout level and monthly family income and also between compassion satisfaction level and area of work of midwife nurses at the 0.05 level of significance.

Conclusion: According to this study, majority of midwives had average level of CF and BO. So, midwives need support and educational training programs to decrease the compassion fatigue and burnout.

Keywords: Compassion Fatigue, Burnout, Compassion Satisfaction, Midwives 


\section{Introduction}

Compassion is defined as "sympathetic consciousness of others' distress together with a desire to relieve it" (The Merriam-Webster Dictionary, 2013). ${ }^{1}$ The phenomenon known as Compassion Fatigue (CF) was first identified by Joinson, in 1992 among nurses. ${ }^{2}$ Compassion fatigue results from taking on the emotional burden of a patient's agony. ${ }^{3}$ Burnout is the state of physical, emotional and mental exhaustion caused by a depletion of a person's ability to cope with one's environment. Compassion satisfaction refers to the fulfillment that an individual derives from his/ her work and form the act of helping itself. ${ }^{4}$

A qualitative non-experimental descriptive survey conducted by Wentzel DL, Brysiewicz P depicts that $55 \%(n=83)$ of nurses had high compassion satisfaction, $61 \%(n=83)$ had average level of burnout and $75 \%(n=83)$ had average level of compassion fatigue. ${ }^{5}$ Another study conducted by Mollart L et al. on 56 Australian midwives, $60.7 \%$ midwives had moderate to high level of emotional exhaustion and $33.3 \%$ midwives were found with burnout. ${ }^{6}$

A descriptive and cross-sectional study was conducted on Turkish 147 midwives who had moderate level of burnout, who had not willingly chosen the profession and worked for economic reasons, who were not pleased with working in their profession had higher mean scores for levels of emotional burnout and depersonalization and a lower mean score for personal accomplishment $(p<0.05){ }^{7}$

Cross-sectional study conducted by Banovcinova, L, Baskova $\mathrm{M}$ revealed sources of occupational stress and their association with burnout in midwives in Slovakia. Convenience sampling method was used to select samples which consisted of 100 Midwives. Expanded Nursing Stress Scale (ENSS) and Maslach Burnout Inventory were employed. Result revealed that the midwives reported high, average and high levels of depersonalization, emotional exhaustion and personal accomplishment, respectively. This study concluded that the interventions must focus on control of stress sources in the working environment as high stress levels often result in burnout of health care workers and change in their attitudes to work, and thus they can negatively influence the care for patients. ${ }^{8}$

A study conducted by Beck $\mathrm{CT}$ et al. revealed the prevalence \& severity of Secondary Traumatic Stress (STS) in Certified Nurse Midwives (CNMs) \&explored their experiences attending traumatic births in America. A convergent, parallel mixed method design was used by sending out e-mail to a total sample of $473 \mathrm{CNMs}$ who completed quantitative portion and 246 who completed the qualitative portion with a link to survey monkey study. In quantitative strand, STS scale was used for collecting data and for the qualitative strand, midwives were asked to describe their experience of attending one or more traumatic births. IBM SPSS 21.0 (version 21.0, Armonk, NV) was used to analyze the quantitative data and Krippendorff content analysis was used for qualitative data. Result reveals $29 \%$ CNMs reported high to severe STS, and $36 \%$ screened for positive mental disorders. This study concluded that midwifery profession should acknowledge STS (Secondary Traumatic Stress) as a professional risk. ${ }^{9}$

A self-administered online survey conducted Fenwick $\mathrm{J}$ et al. revealed personal, professional and workplace factors that contribute to burnout in midwives in Australia. Survey was conducted via an email sent from the Australian College of Midwives between June and July 2014, a total of 1,037 midwives responses were received in which 990 responses were analyzed. The survey included the questionnaire of Copenhagen Burnout Inventory and personal and professional variables. Result revealed that the prevalence of moderate to severe personal $(\mathrm{N}=643$; $64.9 \%$ ) and work-related burnout ( $\mathrm{N}=428 ; 43.8 \%$ ) were high. This study concluded that there should be family-friendly work environment that facilitates work-life balance to reduce the personal and organizational costs of burnout. ${ }^{10}$

A study conducted by Jordan $\mathrm{K}$ et al. revealed level of burnout in midwives working at a maternity unit in South East Queensland, Australia. A self-administered questionnaire was distributed to a total sample of 110 registered midwives in which 58 (52.7\%) midwives completed the package. Questionnaire included the demographic survey and Copenhagen Burnout Inventory. SPSS database version 19 was used to analyze the data. Result revealed that almost $30 \%$ midwives experienced moderate to high levels of burnout and $50 \%$ midwives scored moderate to high for personal burnout. ${ }^{11}$

A meta-analysis study conducted by Ying-Ying $Z$ et al. revealed prevalence of compassion satisfaction, compassion fatigue and burnout in nursing and identified the factors influencing these rates. A sample size was 4054 nurses and data was collected by online survey with Prof-QOL (Professional Quality of Life Scale) scale questionnaire. Results revealed that the prevalence rates of compassion satisfaction, compassion fatigue and burnout were $47.55 \%$, $52.55 \%$ and $51.98 \%$, respectively. This study concluded that in nursing, the prevalence rates of compassions fatigue and burnout are high. Better education \& training may have a moderate effect on compassion fatigue and burnout and could improve the quality of life of nurses. ${ }^{12}$

By rigorous review of literature, it was found that literature was available on compassion fatigue, burnout \& compassion satisfaction among midwives in foreign context but only a few studies were found in Indian context. So, this study aimed to assess prevalence of compassion fatigue, burnout\& compassion satisfaction among staff nurses 
who were working in antenatal, intra-natal and postnatal department in selected hospital of Gautam Buddh Nagar. Objectives of the study were to; assess the prevalence of compassion fatigue, burnout and compassion satisfaction among staff nurses and determine association between compassion fatigue, burnout, compassion satisfaction and selected demographic variables.

\section{Methods}

A non-experimental quantitative research approach with descriptive survey design was adopted and this study was conducted in selected hospitals of Gautam Buddh Nagar. 50 midwives were selected by purposive sampling technique. Data was collected by administering Professional Quality of Life Scale (Pro QOL) by interview technique. Pro QOL Scale is a pre-prepared standardization tool. Also, a demographic proforma was developed which consisted of 10 items. Data collection procedure was carried out from December, 2018 to February, 2019. Data was analyzed using descriptive and inferential statistics.

\section{Result}

According to Table 1, it was found that majority of 36 (72\%) midwives were in age group of $20-30$ years, and female midwife nurses were 46 (92\%). 39 (78\%) midwives were Hindu, 2 (4\%) belonged to Muslim religion and 9 (18\%) were found of Christian religion. 25 (50\%) midwives belonged to nuclear family and 25 (50\%) midwives belonged to joint family. Majority of 26 (52\%) midwives were single or unmarried. Majority of the midwife nurses' 37 (74\%) monthly family income was Rs. 20000-40000 and GNM midwives were $43(86 \%)$, only $1(2 \%)$ midwife nurses were P.B.B.Sc., and 6 (12\%) midwives education level were B.Sc. Nursing. 0-1 year experienced midwife nurses majority were 14 (28\%), more than 1 to 2 years experienced midwife nurses were $10(20 \%)$, and more than 2 to 5 years experienced midwife nurses were 12 (24\%) and remaining more than 5 years experienced midwife nurses were 14 (28\%). Majority of junior staff nurse midwives were 19 (38\%), senior staff nurse midwives were 19 (38\%) also. 25 (50\%) midwives were working in Intra-natal (labor ward).

Table I.Frequency and percentage distribution of midwife nurses as per their demographic variables

\begin{tabular}{|c|c|c|c|}
\hline $\begin{array}{c}\text { S. } \\
\text { No. }\end{array}$ & $\begin{array}{c}\text { Demographic } \\
\text { Variables }\end{array}$ & Frequency & Percentage \\
\hline 1. & \multicolumn{3}{|c|}{ Age group in years } \\
\hline & $20-30$ & 36 & 72 \\
\hline & $31-40$ & 8 & 16 \\
\hline & $41-50$ & 6 & 12 \\
\hline 2. & \multicolumn{3}{|c|}{ Gender } \\
\hline & Male & 4 & 8 \\
\hline
\end{tabular}

\begin{tabular}{|c|c|c|c|}
\hline & Female & 46 & 92 \\
\hline \multirow[t]{4}{*}{3.} & \multicolumn{3}{|c|}{ Religion } \\
\hline & Hindu & 39 & 78 \\
\hline & Muslim & 2 & 4 \\
\hline & Christian & 9 & 18 \\
\hline \multirow[t]{3}{*}{4.} & \multicolumn{3}{|c|}{ Type of Family } \\
\hline & Nuclear & 25 & 50 \\
\hline & Joint & 25 & 50 \\
\hline 5. & \multicolumn{3}{|c|}{ Marital status } \\
\hline & Single & 26 & 52 \\
\hline & Married & 22 & 44 \\
\hline & Divorce/ Separated & 2 & 4 \\
\hline \multirow[t]{5}{*}{6.} & \multicolumn{3}{|c|}{ Family monthly income in Rupees } \\
\hline & $20000-40000$ & 37 & 74 \\
\hline & $40001-60000$ & 9 & 18 \\
\hline & $60001-80000$ & 2 & 4 \\
\hline & More than 80000 & 2 & 4 \\
\hline \multirow[t]{4}{*}{7.} & Education level & & \\
\hline & G.N.M. & 43 & 86 \\
\hline & P. B. B.Sc. Nursing & 1 & 2 \\
\hline & B.Sc. Nursing & 6 & 12 \\
\hline \multirow[t]{5}{*}{8.} & \multicolumn{3}{|c|}{ Years of experience in years } \\
\hline & $0-1$ & 14 & 28 \\
\hline & More than 1 to 2 & 10 & 20 \\
\hline & More than 2 to 5 & 12 & 24 \\
\hline & More than 5 & 14 & 28 \\
\hline \multirow[t]{5}{*}{9.} & \multicolumn{3}{|c|}{ Post/ Designation in profession } \\
\hline & Junior Staff Nurse & 19 & 38 \\
\hline & Senior Staff Nurse & 19 & 38 \\
\hline & Sister in charge & 7 & 14 \\
\hline & Ward in charge & 5 & 10 \\
\hline \multirow[t]{4}{*}{10.} & Area of work & & \\
\hline & Antenatal (OPD) & 4 & 8 \\
\hline & $\begin{array}{c}\text { Intranatal (Labour } \\
\text { room) }\end{array}$ & 25 & 50 \\
\hline & Postnatal ward & 21 & 42 \\
\hline
\end{tabular}

Table 2, shows 43 (86\%) midwife nurses were having average level of compassion fatigue (STS) and 7 (14\%) midwife nurses were having low level of compassion fatigue (STS). Majority of $33(66 \%)$ midwife nurses had average level of burnout, while 17 (34\%) midwife nurses had low level of burnout. Only 16 (32\%) midwife nurses had high level of compassion satisfaction, and while 34 (68\%) midwife nurses had average level of compassion satisfaction. 
Table 2.Frequency and percentage of midwife nurses in different categories of compassion fatigue, burnout and compassion satisfaction

$(n=50)$

\begin{tabular}{|c|c|c|c|c|}
\hline S. No. & Professional quality of life & Score & F & \\
\hline A & Compassion fatigue & & 43 & 86 \\
\hline 1. & Average secondary traumatic stress & Between 23 and 41 & 7 & 14 \\
\hline 2. & Low secondary traumatic stress & 22 or less & 33 & 66 \\
\hline B & Burnout & Between 23 and 41 & 17 & 34 \\
\hline 1. & Average burnout & 22 or less & & \\
\hline 2. & Compassion satisfaction & & 15 & 30 \\
\hline C & High compassion satisfaction & 42 or more & 35 & 70 \\
\hline 1. & Average compassion satisfaction & Between 23 and 41 & 35 \\
\hline 2. & & & 35 \\
\hline
\end{tabular}

Table 3.Association between compassion fatigue and selected demographic variables

$(n=50)$

\begin{tabular}{|c|c|c|c|c|c|c|c|}
\hline \multirow{3}{*}{ S. No. } & \multirow{3}{*}{ Demographic variables } & \multirow{3}{*}{ f } & \multirow{3}{*}{$\%$} & \multicolumn{2}{|c|}{ Compassion fatigue score } & \multirow{3}{*}{ Chi - square } & \multirow{3}{*}{$\begin{array}{c}\text { P-value } \\
(p<0.05)\end{array}$} \\
\hline & & & & >median & $\leq$ median & & \\
\hline & & & & No. & No. & & \\
\hline \multirow[t]{4}{*}{1.} & Age group & & & & & & \\
\hline & $20-30$ years & 36 & 72 & 17 & 19 & \multirow{3}{*}{$4.621(\mathrm{DF}=2)$} & \multirow{3}{*}{$5.99 *$} \\
\hline & $31-40$ years & 8 & 16 & 1 & 7 & & \\
\hline & $41-50$ years & 6 & 12 & 4 & 2 & & \\
\hline \multirow[t]{3}{*}{2.} & Gender & & & & & & \\
\hline & Male & 4 & 8 & 1 & 3 & \multirow{2}{*}{$0.638(\mathrm{DF}=1)$} & \multirow{2}{*}{$3.84^{*}$} \\
\hline & Female & 46 & 92 & 21 & 25 & & \\
\hline \multirow[t]{4}{*}{3.} & Religion & & & & & & \\
\hline & Hindu & 39 & 78 & 16 & 23 & \multirow{3}{*}{$2.686(\mathrm{DF}=2)$} & \multirow{3}{*}{$5.99 *$} \\
\hline & Muslim & 2 & 4 & 2 & 0 & & \\
\hline & Christian & 9 & 18 & 4 & 5 & & \\
\hline \multirow[t]{3}{*}{4.} & Type of family & & & & & & \\
\hline & Nuclear & 25 & 50 & 12 & 13 & \multirow{2}{*}{$0.3246(\mathrm{DF}=1)$} & \multirow{2}{*}{$3.84^{*}$} \\
\hline & Joint & 25 & 50 & 10 & 15 & & \\
\hline \multirow[t]{4}{*}{5.} & Marital status & & & & & & \\
\hline & Single & 26 & 52 & 11 & 15 & \multirow{3}{*}{$2.657(\mathrm{DF}=2)$} & \multirow{3}{*}{$5.99 *$} \\
\hline & Married & 22 & 44 & 9 & 13 & & \\
\hline & Divorce/ Separated & 2 & 4 & 2 & 0 & & \\
\hline \multirow[t]{5}{*}{6.} & Monthly family income & & & & & & \\
\hline & $20000-40000$ & 37 & 74 & 14 & 23 & \multirow{4}{*}{$1.504(\mathrm{DF}=3)$} & \multirow{4}{*}{$7.82^{*}$} \\
\hline & $40001-60000$ & 9 & 18 & 6 & 3 & & \\
\hline & $60001-80000$ & 2 & 4 & 1 & 1 & & \\
\hline & More than 80000 & 2 & 4 & 1 & 1 & & \\
\hline \multirow[t]{2}{*}{7.} & Education level & & & & & & \\
\hline & G.N.M. & 43 & 86 & 20 & 23 & $1.172(\mathrm{DF}=2)$ & $5.99 *$ \\
\hline
\end{tabular}




\begin{tabular}{|c|c|c|c|c|c|c|c|}
\hline & P.B.B.Sc. Nursing & 1 & 2 & 0 & 1 & & \\
\hline & B.Sc. Nursing & 6 & 12 & 2 & 4 & & \\
\hline 8. & Years of experience & & & & & & \\
\hline & $0-1$ year & 14 & 28 & 6 & 8 & \multirow{4}{*}{3.008 (DF=3) } & \multirow{4}{*}{$7.82^{*}$} \\
\hline & More than 1 year to 2 years & 10 & 20 & 6 & 4 & & \\
\hline & More than 2 years to 5 years & 12 & 24 & 3 & 9 & & \\
\hline & More than 5 years & 14 & 28 & 7 & 7 & & \\
\hline \multirow[t]{5}{*}{9.} & $\begin{array}{c}\text { Post/ Designation in } \\
\text { profession }\end{array}$ & & & & & & \\
\hline & Junior Staff Nurse & 19 & 38 & 9 & 10 & \multirow{4}{*}{$1.323(\mathrm{DF}=3)$} & \multirow{4}{*}{$7.82^{*}$} \\
\hline & Senior Staff Nurse & 19 & 38 & 9 & 10 & & \\
\hline & Sister in charge & 7 & 14 & 2 & 5 & & \\
\hline & Ward in charge & 5 & 10 & 2 & 3 & & \\
\hline \multirow[t]{4}{*}{10.} & Area of work & & & & & & \\
\hline & Antenatal (OPD) & 4 & 8 & 2 & 2 & \multirow{3}{*}{$1.677(\mathrm{DF}=2)$} & \multirow{3}{*}{$5.99 *$} \\
\hline & Intranatal (Labour room) & 25 & 50 & 13 & 12 & & \\
\hline & Postnatal ward & 21 & 42 & 7 & 14 & & \\
\hline
\end{tabular}

*Not significant at 0.05 level.

Table 4.Association between burnout and selected demographic variables

\begin{tabular}{|c|c|c|c|c|c|c|c|}
\hline \multirow{3}{*}{ S. No. } & \multirow{3}{*}{ Demographic variables } & \multirow{3}{*}{ f } & \multirow{3}{*}{$\%$} & \multicolumn{2}{|c|}{ Burnout score } & \multirow{3}{*}{ Chi - square } & \multirow{3}{*}{$\begin{array}{c}\text { P-value } \\
\mathrm{p}<0.05 \\
\text { level }\end{array}$} \\
\hline & & & & $>$ median & $\leq$ median & & \\
\hline & & & & No. & No. & & \\
\hline \multirow[t]{4}{*}{1.} & Age group & & & & & & \\
\hline & $20-30$ years & 36 & 72 & 16 & 20 & \multirow{3}{*}{$4.056(\mathrm{DF}=2)$} & \multirow{3}{*}{$5.99 *$} \\
\hline & $31-40$ years & 8 & 16 & 1 & 7 & & \\
\hline & $41-50$ years & 6 & 12 & 1 & 5 & & \\
\hline \multirow[t]{3}{*}{2.} & Gender & & & & & & \\
\hline & Male & 4 & 8 & 3 & 1 & \multirow{2}{*}{$2.86(\mathrm{DF}=1)$} & \multirow{2}{*}{$3.84 *$} \\
\hline & Female & 46 & 92 & 15 & 31 & & \\
\hline \multirow[t]{4}{*}{3.} & Religion & & & & & & \\
\hline & Hindu & 39 & 78 & 15 & 24 & \multirow{3}{*}{$1.252(\mathrm{DF}=2)$} & \multirow{3}{*}{$5.99 *$} \\
\hline & Muslim & 2 & 4 & 0 & 2 & & \\
\hline & Christian & 9 & 18 & 3 & 6 & & \\
\hline \multirow[t]{3}{*}{4.} & Type of family & & & & & & \\
\hline & Nuclear & 25 & 50 & 10 & 15 & \multirow{2}{*}{$0.3472(\mathrm{DF}=1)$} & \multirow{2}{*}{$3.84^{*}$} \\
\hline & Joint & 25 & 50 & 8 & 17 & & \\
\hline \multirow[t]{4}{*}{5.} & Marital status & & & & & & \\
\hline & Single & 26 & 52 & 13 & 13 & \multirow{3}{*}{$5.018(\mathrm{DF}=2)$} & \multirow{3}{*}{$5.99 *$} \\
\hline & Married & 22 & 44 & 5 & 17 & & \\
\hline & Divorce/ Separated & 2 & 4 & 0 & 2 & & \\
\hline
\end{tabular}




\begin{tabular}{|c|c|c|c|c|c|c|c|}
\hline 6. & Family income & & & & & & \\
\hline & $20000-40000$ & 37 & 74 & 13 & 24 & \multirow{4}{*}{$9.542(\mathrm{DF}=3)$} & \multirow{4}{*}{$7.82 * *$} \\
\hline & $40001-60000$ & 9 & 18 & 1 & 8 & & \\
\hline & $60001-80000$ & 2 & 4 & 2 & 0 & & \\
\hline & More than 80000 & 2 & 4 & 2 & 0 & & \\
\hline \multirow[t]{4}{*}{7.} & Education level & & & & & & \\
\hline & G.N.M. & 43 & 86 & 12 & 31 & \multirow{3}{*}{$3.047(\mathrm{DF}=2)$} & \multirow{3}{*}{$5.99 *$} \\
\hline & P.B.B.Sc. Nursing & 1 & 2 & 1 & 0 & & \\
\hline & B.Sc. Nursing & 6 & 12 & 5 & 1 & & \\
\hline \multirow[t]{5}{*}{8.} & Years of experience & & & & & & \\
\hline & $0-1$ year & 14 & 28 & 7 & 7 & \multirow{4}{*}{$4.292(\mathrm{DF}=3)$} & \multirow{4}{*}{$7.82 *$} \\
\hline & More than 1 year to 2 years & 10 & 20 & 4 & 6 & & \\
\hline & More than 2 years to $5 y e a r s$ & 12 & 24 & 5 & 7 & & \\
\hline & More than 5 years & 14 & 28 & 2 & 12 & & \\
\hline \multirow[t]{5}{*}{9.} & Post/ designation in profession & & & & & & \\
\hline & Junior Staff Nurse & 19 & 38 & 9 & 10 & \multirow{4}{*}{$4.521(\mathrm{DF}=3)$} & \multirow{4}{*}{$7.82 *$} \\
\hline & Senior Staff Nurse & 19 & 38 & 5 & 14 & & \\
\hline & Sister in charge & 7 & 14 & 1 & 6 & & \\
\hline & Ward in charge & 5 & 10 & 3 & 2 & & \\
\hline \multirow[t]{4}{*}{10.} & Area of work & & & & & & \\
\hline & Antenatal (OPD) & 4 & 8 & 1 & 3 & \multirow{3}{*}{$4.218(\mathrm{DF}=2)$} & \multirow{3}{*}{$5.99 *$} \\
\hline & Intranatal (Labour room) & 25 & 50 & 6 & 19 & & \\
\hline & Postnatal ward & 21 & 42 & 11 & 10 & & \\
\hline
\end{tabular}

NS*Not significant at 0.05 level; $\mathrm{S}^{* *}$ Significant at 0.05 level.

Table 5.Association between compassion satisfaction and selected demographic variables

$(n=50)$

\begin{tabular}{|c|c|c|c|c|c|c|c|}
\hline \multirow{3}{*}{$\begin{array}{l}\text { S. } \\
\text { No. }\end{array}$} & \multirow{3}{*}{ Demographic variables } & \multirow{3}{*}{ f } & \multirow{3}{*}{$\%$} & \multicolumn{2}{|c|}{ Compassion satisfaction score } & \multirow{3}{*}{ Chi - square } & \multirow{3}{*}{$\begin{array}{c}\text { P-value } \\
p<0.05 \\
\text { level }\end{array}$} \\
\hline & & & & $>$ median & $\leq$ median & & \\
\hline & & & & No. & No. & & \\
\hline \multirow[t]{4}{*}{1.} & Age group & & & & & & \\
\hline & 20-30 years & 36 & 72 & 14 & 22 & \multirow{3}{*}{$2.479(\mathrm{DF}=2)$} & \multirow{3}{*}{$5.99 *$} \\
\hline & $31-40$ years & 8 & 16 & 6 & 2 & & \\
\hline & $41-50$ years & 6 & 12 & 3 & 3 & & \\
\hline \multirow[t]{3}{*}{2.} & Gender & & & & & & \\
\hline & Male & 4 & 8 & 2 & 2 & \multirow{2}{*}{$0.027(\mathrm{DF}=1)$} & \multirow{2}{*}{$3.84 *$} \\
\hline & Female & 46 & 92 & 21 & 25 & & \\
\hline \multirow[t]{4}{*}{3.} & Religion & & & & & & \\
\hline & Hindu & 39 & 78 & 18 & 21 & \multirow{3}{*}{$2.034(\mathrm{DF}=2)$} & \multirow{3}{*}{$5.99 *$} \\
\hline & Muslim & 2 & 4 & 0 & 2 & & \\
\hline & Christian & 9 & 18 & 5 & 4 & & \\
\hline \multirow[t]{2}{*}{4.} & Type of family & & & & & & \\
\hline & Nuclear & 25 & 50 & 9 & 16 & $2.014(\mathrm{DF}=1)$ & $3.84 *$ \\
\hline
\end{tabular}




\begin{tabular}{|c|c|c|c|c|c|c|c|}
\hline & Joint & 25 & 50 & 14 & 11 & & \\
\hline 5. & Marital status & & & & & & \\
\hline & Single & 26 & 52 & 10 & 16 & \multirow{3}{*}{$3.816(\mathrm{DF}=2)$} & \multirow{3}{*}{$5.99 *$} \\
\hline & Married & 22 & 44 & 13 & 9 & & \\
\hline & Divorce/ Separated & 2 & 4 & 0 & 2 & & \\
\hline \multirow[t]{5}{*}{6.} & Family income & & & & & & \\
\hline & $20000-40000$ & 37 & 74 & $?$ & 21 & \multirow{4}{*}{$0.469(\mathrm{DF}=3)$} & \multirow{4}{*}{$7.82 *$} \\
\hline & $40001-60000$ & 9 & 18 & 5 & 4 & & \\
\hline & $60001-80000$ & 2 & 4 & 1 & 1 & & \\
\hline & More than 80000 & 2 & 4 & 1 & 1 & & \\
\hline \multirow[t]{4}{*}{7.} & Education level & & & & & & \\
\hline & G.N.M. & 43 & 86 & 20 & 23 & \multirow{3}{*}{$0.894(\mathrm{DF}=2)$} & \multirow{3}{*}{$5.99 *$} \\
\hline & P.B.B.Sc. Nursing & 1 & 2 & 0 & 1 & & \\
\hline & B.Sc. Nursing & 6 & 12 & 3 & 3 & & \\
\hline \multirow[t]{5}{*}{8.} & Years of experience & & & & & & \\
\hline & $0-1$ year & 14 & 28 & 6 & 8 & \multirow{4}{*}{$1.436(\mathrm{DF}=3)$} & \multirow{4}{*}{$7.82 *$} \\
\hline & More than 1 year to 2 years & 10 & 20 & 3 & 7 & & \\
\hline & More than 2 years to 5 years & 12 & 24 & 6 & 6 & & \\
\hline & Mmore than 5 years & 14 & 28 & 8 & 6 & & \\
\hline \multirow[t]{5}{*}{9.} & Post/ designation in profession & & & & & & \\
\hline & Junior Staff Nurse & 19 & 38 & 6 & 13 & \multirow{4}{*}{$3.821(\mathrm{DF}=3)$} & \multirow{4}{*}{$7.82 *$} \\
\hline & Senior Staff Nurse & 19 & 38 & 10 & 9 & & \\
\hline & Sister in charge & 7 & 14 & 5 & 2 & & \\
\hline & Ward in charge & 5 & 10 & 2 & 3 & & \\
\hline \multirow[t]{4}{*}{10.} & Area of work & & & & & & \\
\hline & Antenatal (OPD) & 4 & 8 & 0 & 4 & \multirow{3}{*}{$8.024(\mathrm{DF}=2)$} & \multirow{3}{*}{$5.99 * *$} \\
\hline & Intranatal (Labour room) & 25 & 50 & 16 & 9 & & \\
\hline & Postnatal ward & 21 & 42 & 7 & 14 & & \\
\hline
\end{tabular}

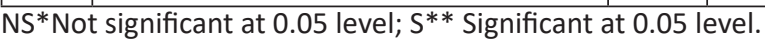

Table 3, depicts that, there was no significant association between the compassion fatigue and age, gender, religion, type of family, marital status, monthly family income, education level, and experience in profession, professional post/ designation, and area of work of the midwife nurses at the 0.05 level of significance.

According to Table 4, there was statistical significant association between the burnout level and monthly family income of midwife nurses at the 0.05 level.

Table 5 shows that, there is significant association between the compassion satisfaction level and area of work of midwife nurses at the 0.05 level of significant.

\section{Discussion}

This study revealed that majority of 43 (86\%) midwife nurses were having average level of compassion fatigue (STS), 33 (66\%) midwife nurses had average level of burnout and only 16 (32\%) midwife nurses had high level of compassion satisfaction. This study revealed that mean score of compassion fatigue was 26.84 , burnout mean score was 24.68 and compassion satisfaction mean score was 38.12. Current study findings are in line with the study investigated by Muliira RS, Ssendikadiwa $\mathrm{VB}^{13}$ to assess professional quality of life of Ugandan midwives, which showed that majority of midwives had average level of compassion satisfaction $68 \%$, burnout $88 \%$, and compassion fatigue (STS) levels $68 \%$. And result found the mean score on the Prof QOL showed compassion satisfaction was 19 , burnout was 36.9 and secondary traumatic stress was 22.9.

In the present study, there was no significant association 
between the burnout level and age, gender, religion, type of family, marital status, education level, and experience in profession, professional post/ designation, and area of work of the midwife nurses at the 0.05 level of significance. Results were partially in agreement with the study conducted by Alparslan O, Doganer G, ${ }^{7}$ which investigated the relationship between burnout related socio-demographic and professional variables and level of burnout of midwives in Sivas in Turkey. Result reveals that Midwives' level of burnout was moderate. There was no association between midwives' burnout and demographic variables like age, marital status, and work area at $p<0.05$ level of significance.

\section{Implications}

This study can be helpful in revision of nursing curriculum for DGNM, B. Sc. Nursing as well as M. Sc. Nursing as areas related to compassion fatigue and burnout may be included so that students cope better with it in future. Midwives who are working in source limited setting of developing countries are exposed to compassion fatigue and burnout and subsequently impacts negatively on their personal and professional life. They may be imparted formal training on how to manage stress issues effectively. There is need for deliberate educational training programs to improve knowledge and skills of midwives to prevent them from secondary traumatic stress. There is need for deliberate training on stress, burnout and trauma, counseling after a stressful encounter and social support services in order to maintain the well-being and professional quality of life of midwives and subsequently the quality of maternal childbirth outcome.

\section{Recommendation}

More research should be conducted about midwives professional quality of life in the future on large sample to validate and generalize the findings. A longitudinal experimental study should be done to assess the effect of support and training on midwives' compassion fatigue and burnout. The hospital authorities must conduct in-service mindfulness based training program for the staff nurses to reduce compassion fatigue.

\section{Conclusion}

On the basis of present study findings, the researcher found that $43(86 \%)$ midwife nurses were having average level of compassion fatigue (STS) and 33 (66\%) midwife nurses had average level of burnout. Midwives need support and educational training programs to decrease the compassion fatigue and burnout and thereby improve satisfaction levels.

\section{Acknowledgement}

Dedicated to My Respected Papa Ji.

Conflicts of Interest: None

\section{References}

1. Compassion and compassion fatigue. Nurse and Midwife Support, 2017. Available from: https:// www.nmsupport.org.au/workplace/compassion-and compassion-fatigue.

2. Harvey J, Burnet J, Kathleen W. The compassion fatigue and resilience connection: a survey of resilience, compassion fatigue, burnout and compassion satisfaction among trauma responders. Int J Emerg Ment Health and Human Resilience 2013; 17(1): 318-26.

3. Jarvis T. Available from: https://nohalfmeasures. blog/2020/05/11/compassion-fatigue/.

4. Stamm BH. Professional Quality of Life: Compassion Satisfaction and Fatigue Version 5 (ProQOL). ProQOL_5_ English_Self-Score, 2009-2012. Available from: https:// www.proqol.org/uploads/ProQOL_5_English_SelfScore_3-2012.pdf.

5. Wentzel DL, Brysiewicz P. A survey of compassion satisfaction, burnout and compassion fatigue in nurses practicing in three oncology departments in Durban, South Africa. Int J of Africa Nurs Sci 2018; 8: 82-6.

6. Mollart L, Skinner VM, Newing $C$ et al. Factors that may influence midwives work-related stress and burnout. Women and Birth 2013; 26(1): 26-32.

7. Alparslan O, Doganer G. Relationship between levels of burnout of midwives who work in Sivas, Turkey province center and identified socio-demographic characteristics. Int J Nurs Midwifery 2009; 1(2): 19-28.

8. Banovcinova L, Baskova M. Sources of work-related stress and their effect on burnout in Midwifery. Procedia Soc and Behav Sci 2014; 132: 248-254.

9. Beck CT, Giudice JL, Gable RK. A mixed method study of STS in certified nurse-midwives: shaken belief in the birth process. J Midwifery Women's Health 2015; 60(1): 16-23.

10. Fenwick J, Lubomsk A, Creedy DK et al. Personal, professional and workplace factors that contribute to burnout in midwives in Australia. US National Library of Medicine National Institutes of Health 2018; 74(4): 852-63.

11. Jordan K, Fenwick J, Slavin V et al. Level of burnout in a small population of Australia midwives. Women Birth 2013; 26(2): 125-132.

12. Ying-Ying Z, Wen-Li H, Wen Q et al. Extent of compassion satisfaction, compassion fatigue and burn out in nursing: A meta-analysis. J Nurs Manag 2018; 26(7): 810-819.

13. Muliira RS, Ssendikadiwa VB. Professional quality of life and associated factors among Ugandan midwives working in Mubende and Mityana rural district. Matern Child Health J 2015; 20(3): 567-576. 